\title{
Características fisicoquímicas e citológicas do líquido sinovial da articulação temporomandibular em eqüinos ${ }^{1}$
}

INDEX TERMS: Equine, synovial fluid, temporomandibular joint.

RESUMO.- Foram estudadas as características fisicoquímicas e citológicas do líquido sinovial da articulação temporomandibular de dez eqüinos hígidos. Verificouse que o líquido é viscoso, amarelo claro a citrino, límpido e livre de partículas à temperatura ambiente. Houve contaminação da amostra por sangue em três amostras que se apresentaram amarelo avermelhadas a vermelhas e de aspecto turvo. A taxa de glicose variou entre $100 \mathrm{e}$ 250 e a concentração protéica não ultrapassou 3,8g/dL. O número médio de células nucleadas foi de 417 células $/ \mu \mathrm{L}$, com predominância de grandes células mononucleares e linfócitos. As mensurações das características

\footnotetext{
${ }^{1}$ Recebido em 2 de março de 2009.

Aceito para publicação em 1 de julho de 2009.

2 Hospital Veterinário de Grandes Animais, Faculdade de Agronomia e Medicina Veterinária, Universidade de Brasília (UnB), Granja do Torto, Brasília, DF 70636-200, Brasil. *Autor para correspondência: robertagodoy@unb.br

${ }^{3}$ Santé Laboratórios, Sociedade Hípica de Brasília, SAIS no.8, Brasília, DF 70602-900, Brasil

${ }^{4}$ Departamento de Clínica, Escola de Veterinária, Universidade Federal de Minas Gerais (UFMG), Av. Antônio Carlos 6627, Belo Horizonte, MG 30123-970, Brasil.
}

pesquisadas no líquido sinovial da articulação temporomandibular de eqüinos são de execução simples e passíveis de implantação na rotina de atendimentos clínicocirúrgicos.

TERMOS DE INDEXAÇÃO: Eqüino, líquido sinovial, articulação temporomandibular.

\section{INTRODUÇÃO}

O líquido sinovial (LS) existente nas cavidades articulares pode ser considerado um fluido especializado que reflete alterações intra-articulares devido a patologias (Van Pelt 1962; 1974). As principais funções do líquido sinovial são lubrificar e nutrir a cartilagem articular, que não possui irrigação nem inervação (Parry 1999). Através da análise do fluido podem-se obter informações sobre a natureza e a extensão das lesões intra-articulares e definir o tratamento e prognóstico, aliada aos sinais clínicos e histórico (Tew \& Hotchkiss 1981, Moyer 1983, Barnabé et al. 2005). Ainda, avaliações seriadas auxiliam na avaliação da resposta à terapia (Kiehl 1997).

A análise do líquido sinovial é de fácil execução e constitui de análise física (cor, volume, turbidez), química (concentração de proteínas totais e formação de coágulo de 
mucina) e citológica (contagem de células nucleadas e análise do esfregaço) (Parry 1999).

A articulação temporomandibular do eqüino é uma diartrose formada, em cada antímero, pelo ramo da mandíbula e a porção escamosa do osso temporal. As partes articulares são de congruências desiguais com a presença de um disco articular entre as superfícies articulares, dividindo a cavidade articular em compartimentos dorsal e ventral. A cápsula articular é reforçada por dois ligamentos, o ligamento lateral e o ligamento caudal (Baker 2002, Moll \& May 2002).

Nos eqüinos, as alterações da articulação temporomandibular podem estar associadas diretamente aos problemas dentários. Sabe-se que anormalidades dentárias levam à inflamação da articulação e conseqüente dor, diminuindo o desempenho do animal, podendo levar a problemas comportamentais. A despeito da sua importância, existem poucos estudos científicos a respeito da ATM em eqüinos, a maioria das informações vem de comparações com estudos desta estrutura em humanos, e ainda não foram estabelecidos padrões de normalidade para o LS existente nesta articulação (Moll \& May 2002, Moura et al. 2004, Schumacher 2006).

Com o advento da odontologia eqüina, é necessário o conhecimento dos parâmetros físico-químicos e citológicos normais do LS da ATM para viabilizar a interpretação de amostras alteradas, pois, como em outras articulações, estas informações são importantes. Assim, o objetivo deste estudo foi analisar as características físicas, químicas e citológicas do líquido sinovial da articulação temporomandibular de eqüinos hígidos.

\section{MATERIAL E MÉTODOS}

Foram utilizados dez eqüinos sem raça definida, adultos e hígidos; sendo três fêmeas e sete machos. Os animais foram selecionados com base na inexistência de doença sistêmica e de ausência de alterações dentárias, após exame da cavidade oral com abre-bocas, fotóforo e espelho.

Os animais foram contidos em um brete de contenção para eqüinos, e tiveram $5,0 \mathrm{~mL}$ de seu sangue venoso colhido por venipunção jugular. $O$ sangue foi acondicionado em tubo contendo EDTA com fluoreto de sódio para determinação de glicose e um tubo sem anticoagulante para determinação de proteína sérica total. Os animais foram sedados com detomidina na dose de $0,03 \mathrm{mg} / \mathrm{kg}$ por via intravenosa para a realização da coleta do líquido sinovial (LS). A região da articulação temporomandibular (ATM) foi submetida à tricotomia e antissepsia de rotina para evitar introdução de contaminantes na articulação.

A punção foi realizada na porção caudal do compartimento dorsal da articulação temporomandibular, segundo a técnica proposta por Rosenstein et al. (2001). O côndilo da mandíbula foi identificado como uma protrusão aproximadamente na porção média , entre a comissura lateral do olho e a base da orelha. $\mathrm{O}$ processo zigomático do osso temporal foi palpado 1-2cm dorsal ao côndilo da mandíbula e uma linha foi traçada entre estas duas estruturas. O local para a punção foi a depressão na área de menor resistência à palpação entre estas duas estruturas e 0,5-1,0 cm caudal à linha imaginária. A agulha era inclinada levemente rostral e ventralmente em uma angulação de aproximadamente $15^{\circ}$ e aprofundada aproximadamente 1,25$3,0 \mathrm{~cm}$. Foi realizada punção com agulha hipodérmica $25 \times 7 \mathrm{~mm}$, com o auxílio de uma seringa de polietileno de $3 \mathrm{~mL}$ (Schumacher 2006). A punção foi feita nas articulações de ambos antímeros de cada animal.

As amostras de LS foram divididas entre um tubo de coleta sem anticoagulante, para dosagem de proteína total pelo método de biureto, e outro com EDTA para as demais determinações, e armazenadas em isopor com gelo, para encaminhamento ao laboratório.

A amostra era primeiramente avaliada macroscopicamente pela sua coloração e aspecto. Posteriormente, o $\mathrm{pH}$ a quantidade de glicose e de proteína foram mensurados através de fita Combur-test ${ }^{\circledR}$ da Roche Diagnostics. A proteína total no LS foi mensurada também através do teste do biureto em um analisador bioquímico automático.

Para as características citológicas do LS, as contagens globais de hemácias e células nucleadas $(\mathrm{CN})$ foram realizadas com hematocitômetro de Neubauer. As contagens diferenciais de $\mathrm{CN}$ foram realizadas nos esfregaços corados com mistura de metanol, May-Grünwald e Giemsa. As amostras foram centrifugadas durante três minutos na velocidade 9 para a confecção das lâminas do sedimento para a análise citológica devido à baixa celularidade encontrada na maioria das amostras de LS que não possuem alteração. Foram contadas 100 células nucleadas de cada lâmina, sendo classificadas como linfócitos, grandes células mononucleares, neutrófilos segmentados e eosinófilos. As grandes células mononucleares constituíramse de monócitos, macrófagos e eventuais células da membrana sinovial.

A glicose plasmática e a proteína total sérica foram analisadas em um analisador bioquímico automático (Labquest).

Os dados foram submetidos à análise estatística descritiva, para estabelecimento de média e desvio padrão dos parâmetros avaliados. Características subjetivas como turbidez e cloração foram submetidas à análise de freqüência. As variáveis paramétricas foram comparadas entre o líquido sinovial da ATM do antímero esquerdo e do antímero direito com o teste $t$ de Student.

\section{RESULTADOS E DISCUSSÃO}

Não houve diferença significativa $(P<0,05)$ entre os antímeros direito e esquerdo quanto às características pesquisadas no líquido sinovial (LS) da ATM.

Obteve-se LS em 95\% (19/20) das punções realizadas na ATM. Em um antímero de um animal não foi coletado nenhuma quantidade de líquido sinovial, aparentemente a articulação estava sem líquido, o que pode caracterizar uma condição inflamatória crônica, ou ser em razão da idade, pois este animal apresentava 11 anos. Segundo Van Pelt (1974) as articulações de animais jovens possuem mais volume de LS.

As amostras analisadas apresentaram volume médio de 2,0 $\pm 0,9 \mathrm{~mL}$. O volume é compatível com o tamanho da ATM e com o fato de não haver comunicação entre seus compartimentos dorsal e ventral. Van Pelt (1974) relata que o volume total do líquido sinovial aspirado de qualquer articulação geralmente varia em proporção ao tamanho articular e a comunicação com outra articulação. As 


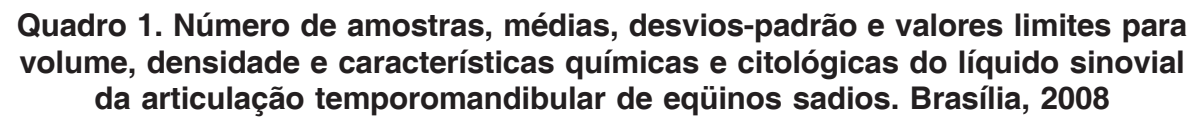

\begin{tabular}{l}
\hline \multicolumn{1}{c}{ Variável } \\
\hline Volume $(\mathrm{mL})$ \\
$\mathrm{pH}$ \\
Densidade \\
Glicose $(\mathrm{mg} / \mathrm{dL})$ \\
Proteínas totais - Método do Biureto(g/dL) \\
Proteínas totais - Fita $(\mathrm{g} / \mathrm{dL})$ \\
Hemácias (células/ $\mu \mathrm{L})$ \\
Células nucleadas (células/ $\mu \mathrm{L})$ \\
Neutrófilos segmentados $(\%)$ \\
Linfócitos (\%) \\
Grandes células mononucleares $(\%)$ \\
Eosinófilos (\%)
\end{tabular}

patologias articulares geralmente acarretam em alterações de volume e composição do líquido sinovial. Van Pelt (1974) e Parry (1999) afirmaram que o volume de LS obtido varia de acordo com o tamanho da articulação. Parry (1999) estudando várias articulações de cães obteve volume variando de 0,01-1,0mL.

Nas 11 amostras colhidas houve predomínio do aspecto amarelo claro em $47,4 \%$ dos animais (9/19), límpido e ausência de partículas em suspensão. Três amostras $(15,8 \%)$ foram contaminadas com sangue durante a punção da ATM, apresentando coloração amarelo-avermeIhada a vermelha e aspecto turvo, o que, segundo Barnabé et al. (2005), é comum na punção de líquido sinovial, em conseqüência da ruptura de capilares subsinoviais e essa pôde ser confirmada pela inexistência de amostras com coloração alterada no início da punção e coloração amarelo-claro após sua centrifugação, ou seja ausência de xantocromia. Segundo Brannan et al. (2006), em geral, o aspecto límpido do líquido está relacionado com a baixa contagem de leucócitos da amostra, pois esta quanto estiver acima dos níveis normais levará a um aspecto semiturvo a turvo do LS.

Volume, densidade e características químicas e citológicas do LS da ATM dos eqüinos encontram-se no Quadro 1.

Em todas as amostras, na análise com a fita, a concentração de proteína total foi igual a $2,0 \mathrm{~g} / \mathrm{dL}$, pois este é o valor limítrofe para a aferição deste parâmetro com a fita. Sugere-se então que a análise de proteína total seja realizada pelo método do biureto para uma maior acurácia.

Quando analisadas pelo método do biureto as amostras de LS, apresentaram uma média de $2,73 \mathrm{~g} / \mathrm{dL}$ de proteínas totais, corroborando os achados de Parry (1999) em cães, que obteve entre 1,8 e $4,8 \mathrm{~g} / \mathrm{dL}$. No entanto, no que se refere à proteína total, os achados neste estudo discordam de Barnabé et al. (2005) que relataram uma concentração protéica de $0,60-1,75 \mathrm{~g} / \mathrm{dL}$ no LS de eqüinos. Porém esta baixa concentração de proteína encontrada por Barnabé et al. (2005) pode ser reflexo do jejum de 24 horas, ao qual os eqüinos foram submetidos antes da coleta. Valores aumentados de proteína são encon-

$\begin{array}{ccc}\begin{array}{c}\text { Número de } \\ \text { amostras }\end{array} & \text { Média } \pm \text { erro-padrão } & \text { Valores limite } \\ 19 & 2,0 \pm 0,9 & 0,5-3,0 \\ 19 & 8,56 \pm 0,42 & 8-9 \\ 19 & 1.020,61 \pm 3,84 & 1.012-1.027 \\ 19 & 108,33 \pm 35,36 & 100-250 \\ 12 & 2,73 \pm 0,98 & 2,13-3,8 \\ 19 & 2,0 \pm 0 & 2,0 \\ 18 & 1.282 \pm 2.334 & 0-57.000 \\ 18 & 417 \pm 239 & 70-870 \\ 16 & 8,33 \pm 9,57 & 0-36 \\ 16 & 60,5 \pm 18,43 & 10-90 \\ 16 & 26,22 \pm 22,86 & 0-90 \\ 16 & 0,33 \pm 0,77 & 0-2\end{array}$

trados em processos inflamatórios, bem como a presença de proteínas maiores, como o fibrinogênio, entram no líquido sinovial.

A concentração sérica média de proteínas totais foi de $8,63 \pm 0,72 \mathrm{~g} / \mathrm{dL}$, sendo que a proteína, avaliada pelo método do Biureto, no LS correspondeu, em média, a $31,63 \%$ da concentração plasmática, concordando com os achados de Curtiss (1964), van Pelt (1974), Levick (1987) e Rasera (2007). Porém a proporção de proteína total no LS em relação à sérica diferem dos achados de Barnabé et al. (2005) que obtiveram uma concentração de proteína no LS cerca de $16,2 \%$ em relação à sérica, não indicando porém patologias. Durante as inflamações articulares a proteína no LS tende a estar proporcionalmente aumentada, em relação à proteína sérica, pelo aumento da permeabilidade da membrana sinovial às macromoléculas (Rasera 2007).

A concentração média de glicose no LS se mostrou próxima ao limite superior em relação à concentração plasmática considerada normal $(75-115 \mathrm{mg} / \mathrm{dL})$ para a espécie (Kaneko et al. 1997), conforme descrito por Curtiss (1964); Van Pelt (1974) e Barnabé (2001). Quando comprada à concentração plasmática de glicose $(98,34 \pm 19,61 \mathrm{mg} / \mathrm{dL})$, a concentração no LS foi $10 \%$ superior, porém sem diferença significativa, concordando com os achados de Van Pelt (1974) e Barnabé et al. (2005) que também encontraram valores similares entre a concentração de glicose plasmática e no LS. De acordo com Mahaffey (2002), em condições normais existe um equilíbrio de glicose entre o sangue e LS, obtido pela livre difusão desta molécula através da membrana sinovial.

Neste ensaio, o número de hemácias nas amostras de LS variou consideravelmente, devido à contaminação de amostras de LS no momento da punção, ainda que tenha sido notada visualmente em apenas três amostras. Os achados referentes às hemácias no LS são compatíveis com os achados de Barnabé et al. (2005) no LS de eqüinos.

O valor médio da contagem global de células nucleadas (CN) manteve-se entre aqueles considerados normais para o LS (Van Pelt 1967, 1974, Tew \& Hotchkiss 1981, 
Malark et al. 1991, Barnabé et al. 2005). Os linfócitos predominaram, seguidos pelas grandes células mononucleares, neutrófilos segmentados e eosinófilos, discordando dos achados de Barnabé et al. (2005) que obtiveram uma maior proporção de grandes células mononucleares, seguidas por linfócitos, neutrófilos segmentados e eosinófilos. No entanto, os resultados foram condizentes com Van Pelt (1974) e Rasera (2007) que relataram predominância de células mononucleares, ou seja linfócitos e monócitos. Estas variações podem indicar que cada articulação possui características individuais, salientando a importância de se obter parâmetros de normalidade especificamente.

A contagem de células nucleadas em líquido sinovial normal varia conforme a articulação, mas o normal é se apresentarem abaixo 3.000/ $\mathrm{LL}$ (Parry 1999). Neste estudo, todas amostras apresentaram valores inferiores a este limite, podendo ser consideradas amostras de articulações saudáveis ( $70-870$ células/ $\mu \mathrm{L})$. Segundo Brannan et. al (2006), a contagem global de células é um exame importante para a classificação de processos articulares como inflamatórios ou não-inflamatórios.

Algumas amostras apresentaram inversão de predominância entre as grandes células mononucleares e os linfócitos, mas ambos os tipos celulares sempre foram os mais abundantes. Barnabé et al. (2005) também observaram esta característica e atribuíram à influência que a concentração e o grau de polimerização da molécula de ácido hialurônico exercem sobre a migração linfocitária para a cavidade sinovial.

De acordo com Van Pelt (1962), as características fisico-químicas e citológicas do líquido sinovial normal podem ser empregadas para avaliar as várias artrites, estabelecer diagnósticos precoces e prognósticos, determinar a resposta articular às terapias. As alterações patológicas decorrentes das artrites e sinovites, provavelmente, se manifestam precocemente no LS, como descrito nos processos articulares por Tulamo et al. (1989), alterando sua composição físico-química e citológica antes que outros sintomas clínicos apareçam. Assim, uma vez conhecidos os valores de referência para o LS da ATM dos eqüinos, sua análise provê informações que agilizam o diagnóstico (Barnabé 2001, Barnabé et al. 2004), podendo ajudar no estabelecimento do grau de severidade das lesões associadas a essas estruturas, propiciando elementos dinâmicos para reavaliações de casos clínicos e prognóstico mais acurado.

\section{CONCLUSÕES}

As mensurações das características pesquisadas no líquido sinovial (LS) da ATM de eqüinos são de execução simples e passíveis de implantação na rotina de atendimentos clínico-cirúrgicos, podendo trazer contribuição significativa ao diagnóstico, tratamento e prognóstico de animais com alterações patológicas da ATM. A contaminação de amostras de LS com pequena quantidade de sangue não ocasiona prejuízos a sua análise.
O valor médio da contagem global de células nucleadas (CN) foi de $417 \pm 239$ células/ $\mu \mathrm{L}$. Os linfócitos predominaram, seguidos pelas grandes células mononucleares, neutrófilos segmentados e eosinófilos.

$\mathrm{Na}$ coleta de LS as amostras devem ser divididas em tubos com e sem EDTA para viabilizar uma melhor mensuração da concentração de proteínas totais pelo método do biureto.

Agradecimentos.- À FAPDF pelo apoio financeiro para execução da pesquisa (Proc.193.000.468/2008).

\section{REFERÊNCIAS}

Baker G.J. 2002. Equine temporomandibular joints (TMJ): Morphology, function and clinical disease Proc. $48^{\text {th }}$ Annual Convention of the American Association of Equine Practitioners, p.442-447. Disponível em: http://www.ivis.org/proceedings/AAEP/2002/contents.pdf (Acesso 20 nov. 2008).

Barnabé P.A. 2001. Estudo das características físico-químicas e citológicas do líquido sinovial da bainha tendínea digital de eqüinos. Dissertação de Mestrado em Cirurgia Veterinária, Universidade Estadual Paulista, Jaboticabal, SP. 96p.

Barnabé P.A., Cattelan J.W. \& Cadioli FA. 2004. Anatomofisiologia da bainha tendínea sinovial digital dos eqüinos. Ciência Rural 34(2):619623.

Barnabé P.A., Cattelan J.W., Cadioli F.A. \& Godoy R.F. 2005 Características físico-químicas e citológicas do líquido sinovial da bainha tendínea digital de eqüinos. Arq. Bras. Med. Vet. Zootec. 57(3):288294.

Brannan S.R. \& Jerrard D.A. 2006. Synovial fluid analysis. J. Emerg. Med. 30(3):331-339.

Curtiss P.H. 2006. Changes produced in the synovial membrane and synovial fluid by disease. J. Bone Joint Surg. Am. 46(4):873-888.

Kaneko J.J., Harvey J.W. \& Bruss M.L. 1997. Clinical Biochemistry of Domestic Animals. $5^{\text {th }}$ ed. Academic Press, London, p.885-906.

Kiehl A.R. 1997. Synovial fluid analysis. Proc. $14^{\text {th }}$ American College of Veterinary Internal Medicine Forum, Lake Buena Vista, FL.

Mahaffey E.A. 2001. Synovial fluid, p.163-170. In: Cowell R.L. \& Tyler R.D. (Eds), Diagnostic Cytology and Hematology of the Horse. 2nd ed. Mosby, St Louis.

Malark J.A., Nixon A.J., Skinner K.L. \& Mohammed H. 1991. Characteristics of digital flexor tendon sheath fluid from clinically normal horses. Am. J. Vet. Res. 52(8):1292-1294.

Moll H.D. \& May K.A. 2002. A review of conditions of the equine temporomandibular joint. Proc. $48^{\text {th }}$ Annual Convention of the American Association of Equine Practitioners, p.240-243. Disponível em: http:/ /www.ivis.org/proceedings/AAEP/2002/contents.pdf (Acesso 20 nov. 2008).

Moura A.G., Rodrigues D.A., Silva M.R.M.A., Bernardino Jr R. \& Severino R.S. 2004. Anatomia comparada da articulação temporomandibular. Biosc. J. 20(3). Disponível em: www.biosciencejournal.ufu.br/include/ getdoc. .php id $=660 \&$ article=202\&mode $=$ pdf (Acesso 18 nov. 2008).

Moyer W. 1983. Clinical use of synovial fluid analysis. Proc. $28^{\text {th }}$ Annual Convention of the American Association of Equine Practitioners, p.129135.

Parry B.W. 1999. Synovial fluid, p.104-119. In: Cowell R.L., Tyler R.D. \& Meinkoth J.H. (Eds), Diagnostic Cytology and Hematology of the Dog and Cat. 2nd ed. Mosby, St Louis.

Rasera L. 2004. Influência da atividade física sobre a articulação metacarpofalangeana de cavalos de pólo. Tese de Doutorado em Clínica Veterinária, USP, São Paulo. 158p.

Rosenstein D.S., Bullock M.F., Ocello P.J. \& Clayton H.M. 2001. 
Arthrocentesis of the temporomandibular joint in adult horses. Am. J. Vet. Res. 62(5):729-733.

Schumacher J. 2006. The equine temporomandibular joint. Proc. AAEP Focus Meeting on Equine Dentistry, American Association Equine Practitioners, Indianapolis. (CD-ROM)

Tew W.P. \& Hotchkiss R.N. 1981. Synovial fluid analysis and equine joint disorders. J. Eq. Vet. Sci. 1:163-170.

Tulamo R.M., Bramlage L.R. \& Gabel A.A. 1989. Sequential clinical and synovial fluid changes associated with acute infectious arthritis in the horse. Eq. Vet. J. 21:325-331.

Van Pelt R.W. 1962. Properties of equine synovial fluid. J. Am. Vet. Med. Assoc. 141:1051-1061.

Van Pelt R.W. 1967. Characteristics of normal equine tarsal synovial fluid. Can. J. Comp. Med. Vet. Sci. 31(12):342-347.

Van Pelt R.W. 1974. Interpretation of synovial fluid findings in the horse. J. Am. Vet. Med. Assoc. 165:91-95. 